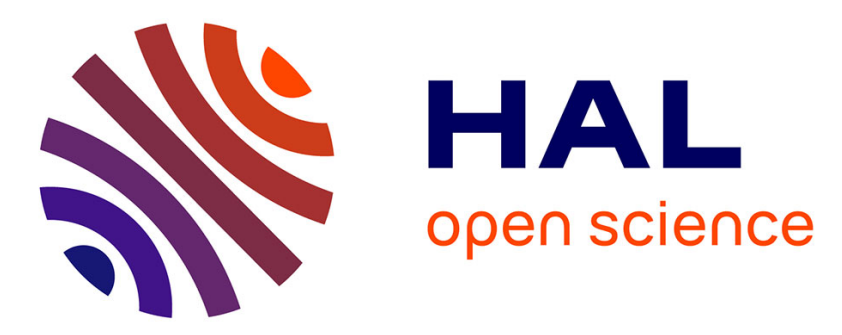

\title{
Mesure de l'intensité en valeur absolue du rayonnement $\gamma$ dur de AcC
}

\author{
Nicolas C.M. Marty
}

\section{To cite this version:}

Nicolas C.M. Marty. Mesure de l'intensité en valeur absolue du rayonnement $\gamma$ dur de AcC. J. Phys.

Radium, 1944, 5 (12), pp.276-278. 10.1051/jphysrad:01944005012027601 . jpa-00233893

\section{HAL Id: jpa-00233893 https://hal.science/jpa-00233893}

Submitted on 1 Jan 1944

HAL is a multi-disciplinary open access archive for the deposit and dissemination of scientific research documents, whether they are published or not. The documents may come from teaching and research institutions in France or abroad, or from public or private research centers.
L'archive ouverte pluridisciplinaire HAL, est destinée au dépôt et à la diffusion de documents scientifiques de niveau recherche, publiés ou non, émanant des établissements d'enseignement et de recherche français ou étrangers, des laboratoires publics ou privés. 


\title{
MESURE DE L'INTENSITÉ EN VALEUR ABSOLUE DU RAYONNEMENT ‘ DUR DE Ac $C$
}

Par Mme N. MARTY.

Laboratoire de Synthèse Atomique d’Ivry.

\begin{abstract}
Sommaire. - On a mesuré l'intensité en valeur absolue du rayonnement $\gamma$ de o,829 $\mathrm{M} \mathrm{eV}$ émis par Ac $C$ en utilisant un compteur Geiger-Müller à paroi épaisse; on décrit, à ce propos, une méthode de calcul de l'efficacité d'un compteur pour un rayonnement d'énergie donnée; le nombre de désintégrations de la source a été mesuré par la méthode du grand condensateur à travers $\mathrm{I} \mathrm{cm}$ de plomb et le nombre de rayons $\gamma$ émis, par la méthode d'absorption à travers des écrans de $\mathrm{Cu}, \mathrm{Pb}, \mathrm{Al}$; on obtient la valeur de $5, y_{4}$ I 2 quanta pour roo désintégrations.
\end{abstract}

Introduction. - De nombreuses méthodes ont été suivies pour déterminer l'intensité des rayonnements $\gamma$. L'utilisation des compteurs GeigerMüller pour les quanta de grande énergie pose le problème de l'efficacité relative à la longueur d'onde du rayonnement étudié [r]; c'est cette méthode que nous avons appliquée à l'étude du rayonnement de o,829 MeV, émis par Ac C et nous avons calculé directement l'efficacité correspondante du compteur.

Principe de la méthode. - Pour connaître le nombre de quanta $N_{q}$ d'une énergie donnée, émis par désintégration, il faut déterminer, d'une part l'intensité de la source, c'est-à-dire le nombre de désintégrations par seconde, ceci a été effectué à 
l'Institut du Radium par la méthode du grand condensateur à travers $\mathrm{I} \mathrm{cm}$ de plomb, et, d'autre part, le nombre $I$ de photons $\gamma$ de cette énergie émis par la source pendant le même temps. $I$ a été mesuré par l'intermédiaire des électrons Compton formés par les rayons $\gamma$ dans la paroi d'un compteur Geiger-Müller.

Soit $i=\frac{I}{4 \pi r^{2}}$ le nombre de quanta qui tombent sur ${\mathrm{I} \mathrm{cm}^{2}}^{2}$ de surface normale à la direction du faisceau incident à la distance $r$ de la source à laquelle se trouve le compteur, et $n_{0}$ l'intensité misurée au compteur en nombre de coups; nous définirons l'efficacité du compteur par la relation $e=\frac{n_{0}}{i}$; c'est le nombre de décharges qui se produisent au compteur lorsqu'il arrive un quantum par unité de surface normale à la direction du faisceau incident. Nous sommes donc ramenés à déterminer $n_{0}$ et $e$.

$n_{0}$ est obtenu par extrapolation de la courbe d'absorption avec écrans de $\mathrm{Cu}, \mathrm{Pb}, \mathrm{Al}$, pour une épaisseur nulle d'écrans; on a interposé des épaisseurs de métal telles que les rayonnements de plus faible énergie que $0,829 \mathrm{MeV}$ soient totalement absorbés, $e$ a été calculé pour un compteur GeigerMüller à paroi de cuivre de $0,04 \mathrm{~cm}$ d'épaisseur, 4, $8 \mathrm{~cm}$ de hauteur, 2,2 $\mathrm{cm}$ de diamètre, muni d'un fil de tungstène de o,or $\mathrm{cm}$ de diamètre et rempli de mélange argon-acétone sous la pression de $?_{1} \mathrm{~cm}$.

Considérons $m$ quanta arrivant sur $\mathrm{I} \mathrm{cm}^{2}$ de surface normale au faisceau incident; après la traversée d'une épaisseur $x$ de matière pour laquelle le coefficient d'absorption relatif à l'énergie considérée est $\mu$, il n'y a plus que $m e^{-\mu x}$ quanta, le reste $m\left(\mathrm{I}-e^{-\mu x}\right)$ quanta s'étant transformé en électrons Compton (c'est le seul effet qui intervienne dans le cas actuel) et en rayons $\gamma$ de moindre énergie pour lesquels la probabilité d'une nouvelle transformation est tout à fait négligeable. Sur ces $m\left(\mathrm{I}-e^{-\mu \cdot x}\right)$ électrons, seuls $k m\left(\mathrm{I}-e^{-\mu . x}\right)$ pénètrent à l'intérieur du compteur et provoquent la décharge; le coefficient $k$ dépend du lieu de formation de l'électron et de la direction suivant laquelle il est projeté. L'épaisseur de la paroi du compteur a été calculée de façon que les électrons formés à l'extérieur de celui-ci ne puissent la traverser.

Pour la commodité des calculs, la paroi du cylindre a été découpée en éléments de volume à l'aide de plans diamétraux et de cylindres concentriques déterminant ainsi, en section droite, un certain nombre de couronnes concentriques et de secteurs (cf. fig. I) le coefficient $k$, calculé pour le point milieu de chaque élément, constitue une approximation suffisante de la valeur moyenne relative à l'élément.

Soit $h$ la hauteur du compteur, $l_{p}$ la projection de l'arc limitant le secteur $p$ sur la perpendiculaire à la direction du faisceau incident dans le plan de section droite, $x_{p q}$ l'épaisseur de la couronne $q$ au milieu de l'élément $p q$, $\mu$ le coefficient d'absorption relatif à l'énergie $0,829 \mathrm{MeV}$, pour $m$ quanta tombant par centimètre carré de surface normale à la direction du faisceau incident, il en tombe $m h l_{p}$ sur l'élément $p q$, il y a $m h l_{\nu}\left(\mathrm{I}-e^{-\mu . x_{p q}}\right)$ électrons Compton

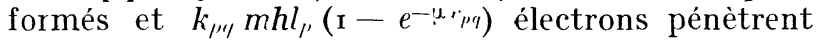

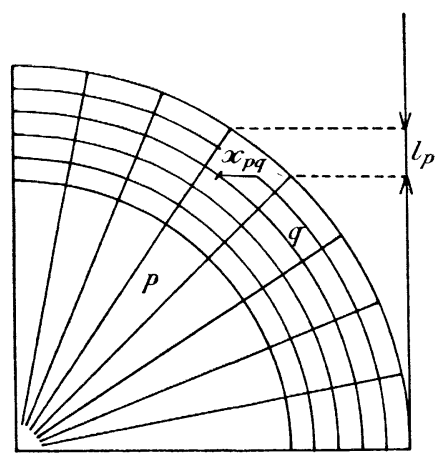

Fig. I.

à l'intérieur du compteur. Les coefficients $k_{p q}$ ont été calculés en tenant compte du nombre d'électrons projetés sous un angle donné [2], de l'énergie des électrons en fonction de la direction d'émission, et de l'épaisseur de métal traversée en fonction de l'énergie des électrons [3]. La longueur $l_{p}$ est à très peu près invariable pour tous les éléments d'un même secteur. La contribution du secteur $p$ en électrons effectivement comptés est donc

$$
m h l_{p} \sum_{q} k_{p q}\left(\mathrm{I}-e^{\left.-u x_{p q}\right)}\right.
$$

et le nombre d'électrons qui proviennent de l'ensemble de la paroi du compteur et déterminent la décharge est

$$
m h \sum_{p} l_{p} \sum_{q} k_{p y}\left(\mathrm{I}-e^{\left.-\mu x_{p q}\right)} .\right.
$$

Ramenons le calcul au cas d'un électron tombant par centimètre carré de surface normale à la direction du faisceau incident, nous obtenons l'efficacité $e$ du compteur

$$
e=h \sum_{p} l_{p} \sum_{q /} k_{p q}\left(\mathrm{I}-e^{\left.-\mu . x_{p q}\right)}\right.
$$

s'il en arrive $i$, il y a $n_{0}$ décharges au compteur

$$
n_{0}=i e
$$

Pour le compteur utilisé

$$
e=6.10^{-2} \pm 0,6 .
$$

La faible précision avec laquelle sont connues les relations parcours-énergie et le pourcentage des électrons projetés sous un angle donné ne permet pas 
d'obtenir la valeur de $e$ avec une précision supérieure à ıo pour ıoo.

Dispositif expérimental (fig. 2). - Devant la source, entourée d'une protection de plomb, se trouve un canaliseur en plomb de $20 \mathrm{~cm}$ de long,
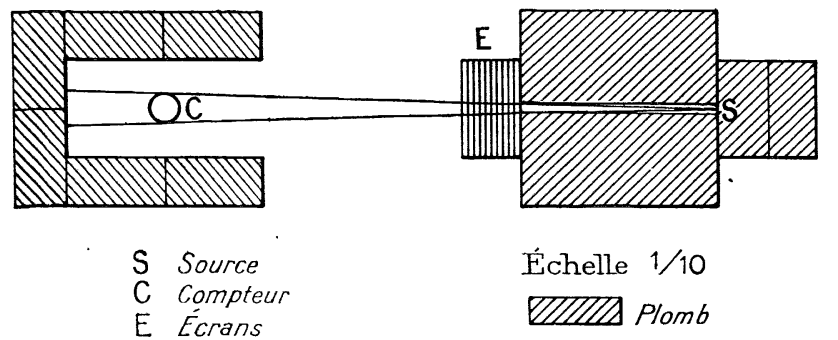

Fig. 2.

$5 \mathrm{~cm}$ de haut, I, $4 \mathrm{~cm}$ de large à l'extrémité duquel sont placés les écrans; le compteur, protégé contre le rayonnement extérieur par une enceinte en briques de plomb, est relié à un amplificateur suivi d'un numérateur type Berthelot d'après le montage habituellement utilisé au Laboratoire de Chimie nucléaire du Collège de France.

Résultats. - L'intensité de la préparation d'Ac a été trouvée égale à $\mathrm{r}, 3$ I millicuries en considérant que I mg de Ra est équivalent en rayons $\gamma$ à travers $\mathrm{I} \mathrm{cm}$ de plomb à $28^{-}$millicuries d'Ac. Donc, le nombre de désintégrations de la source par minute est de 2,9,10. La distance source-compteur $r$ était de $56 \mathrm{~cm}$, le mouvement propre de 4,5 coups par minute. Dans le tracé des courbes d'absorption relatives aux trois sortes d'écrans $\mathrm{Cu}, \mathrm{Pb}, \mathrm{Al}$, si l'on porte en abscisse l'épaisseur des écrans et en ordonnée le logarithme du nombre de coups, en tenant compte de l'imprécision sur les ordonnées, on pouvait mener par les points expérimentaux les droites de pente théorique (calculées d'après les formules de Klein et Nishina pour l'effet Compton et de Hulme pour l'effet photoélectrique); d'où l'on déduisait l'intensité $n_{0}$ correspondant à une épaisseur nulle d'écrans.

Les résultats obtenus sont résumés dans le tableau suivant :

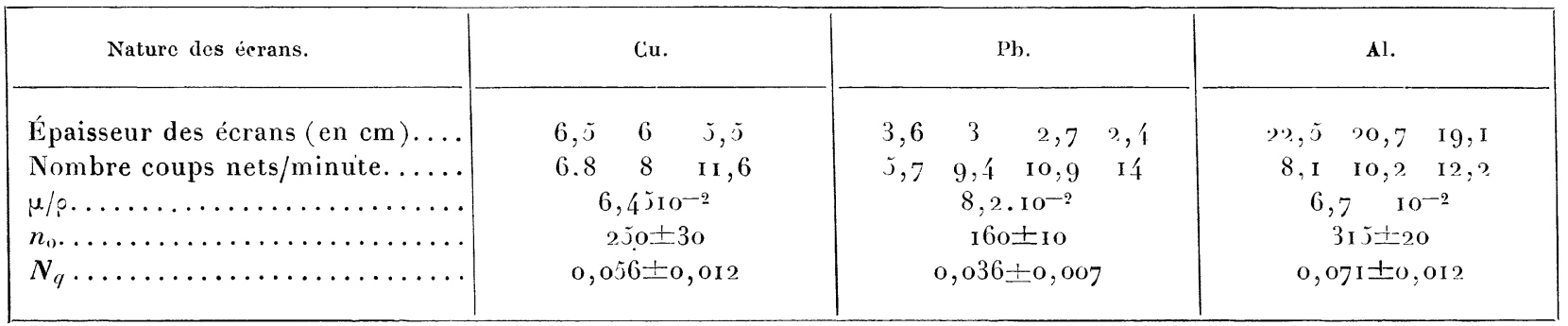

C'est dans le cas des mesures faites avec les écrans de $\mathrm{Cu}$ que le fonctionnement du compteur offrait le maximum de stabilité. Il convient donc d'attribuer le plus de valeur à ces mesures. Les différences entre les résultats obtenus avec les trois sortes d'écrans peuvent s'expliquer par la diffusion des électrons à travers des épaisseurs d'écran différentes entraînant des distances différentes entre le dernier écran et le compteur. Malgré cela, l'accord est satisfaisant avec les résultats obtenus par Savel et Bachelet [4] d'après lesquels l'intensité du groupe de rayons $\gamma$ d'environ $0,9 \mathrm{M} \mathrm{eV}$ émis par Ac en présence de tous ses dérivés serait de $5 \pm 0,5$ quanta pour i oo désintégrations.
Ces résultats viennent confirmer les conclusions de J. Surugue [j] suivant lesquelles le rayonnement $\gamma$ de Ac C de o,829 M eV serait un rayonnement quadripolaire; en effet, dans ce cas, son intensité, calculée à partir des coefficients de conversion interne serait de o, 33 quanta par désintégration, alors qu'elle serait supérieure à 0 ,-4 quanta par désintégration dans le cas d'un rayonnement dipolaire.

Je tiens à remercier ici M. Joliot grâce à qui j'ai $\mathrm{pu}$ entreprendre ce travail et M. Surugue qui m'en a donné le sujet et m'a conseillée et dirigée tout au long de son exécution avec la plus grande bienveillance.

BIBL IOGRAPHIE.

[1] Von Droste, Zeits. für physik, 100, ig36, p. 529.

[2]. Kahan, $J$. de Physique, 10, 1939, p. 430.

[3] Schonlaxd, Proc. Roy. Soc., 108, I925, p. I 87.
[4] Savel et Bachelet, Cahiers de Physique, 19, i9ł' p. јт. [5] Surugue, J. de Physique, 1942, p. - I. 